\title{
Visualizing the Generated Design Process with Dynamic Graph
}

\section{Wei-Hsien Lan, Teng-Wen Chang}

\author{
National Yunlin University of Science and Technology \\ Email:blue@softlab.tw,tengwen@yuntech.edu.tw
}

\begin{abstract}
Generally speaking, we can consider that visualize something or certain process as a kind of simulate process. Therefore, this research regards the visualized form transformation during the generative design process as a kind of imitated process. The design process is a continuous decision-making action, however, the way that designers execute design is usually by way of from state to state during design decision-making process and it let designers lose some important information such as glance of design or form generative process. Those design information that lost and without recorded are significant information during design thinking and process.

This research takes watch design as an example. Base on the theory of Wassily Kandinsky the rule of Genetic Algorithms, the system decodes basic forms of a watch and then encodes by forms and texture. After encoding, the system generates a computer-aided watch forms design prototype by way of crossover of Genetic Algorithms and collects all data during design process. Furthermore, by using these data to bring out Dynamic Graphic during form transformation based on state space and to probe whether or not it can help designers.
\end{abstract}

Keywords: visualize the design process, state space search, Generative Design, Dynamic Graph 


\section{INTRODUCTION}

\subsection{General background information}

Generally speaking, the thinking of designers is often jumped and can be pictured although it is a continuous decision-making action. During decision-making process, the designer executes design by way of way of state to state yet it brings design discontinuousness. The discontinuousness causes designer loses some design information.

Among these continuous actions, some latent disguised states are possibly close to designer's original thinking yet belong to unobvious states. Therefore, find out how to make states bring continuous observations is possibly to seek any continuous point and extend downward amid continuous process. It will become the start point of a new form development as well as a new decision point. From derivation graphs point of view to treat about the relationship between state to state (FIGURE 1), derivation graphs reveal piecemeal situation message and it seems can represent dynamic design process.

Among the researches focus on design thinking and process, protocol analysis is the most use research method to get information during aided design process. However, it just reveals and records inner design thinking. This research aims at the outer form transformation during design process, and therefore protocol analysis is not suitable for the research. So, the research is based on the theory of Wassily Kandinsky- triangle, square and circle are classical form elements and are original source of form as well. Base on the design concept that three basic forms can achieve all kinds of form transformation, we are able to build a prototype that automatically generates the geometric watch design in accordance with genetic algorithm. By using this system to collect and research data that result from design process.

\subsection{Research purpose}

The research expands generative design of watch by way of the operation of the prototype. By this system, the research collects data during design process and visualizes the design process by STATE SPACE SEARCH. Furthermore, the research investigates whether or not the dynamic graphic can help designers.

\section{LITERATURE REVIEW}

\subsection{Design process}

Although there are large amount of variable and possibilities, the design still can be induced to several indifferent design models and present the common behavior logical structure based on some common design actions:

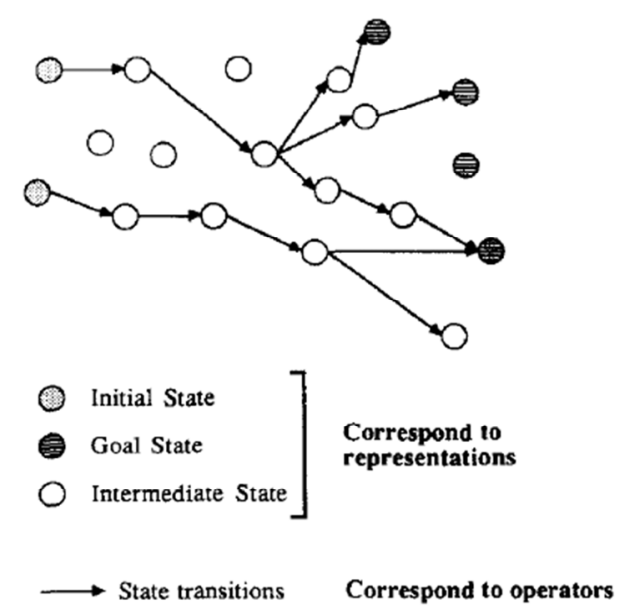

Figure 1. A generic diagram for search [Woodbury, 1991]

Asimow's model (Asimow, 1962) : The structure of design process consists of continuous behavior of the vertical structure and manufacturing cycle of horizontal structure. The vertical structure of design behavior is the process from abstract to concrete; however, along with the situation of design information, the horizontal structure shows a flowing situation of Analysis $\rightarrow$ Synthesis $\rightarrow$ Evaluate $\rightarrow$ communication (FIGURE 2).

Archer' s design process phase model(Archer, 1965) : From design operation point of view, design is seen as a continuous phase-type action. Design has its origin and common task type, that' $s$ why design is described as an ordinary type. Design process is a cycled feedback loop and presents the relationship between actions. (FIGURE 3)

“Analysis $\rightarrow$ Synthesis $\rightarrow$ Evaluate" (Alexander, 1964) $:$ (after adding $\ulcorner$ Evaluate $\lrcorner$, the derivation of this model becomes "Aalysis $\rightarrow$ Synthesis $\rightarrow$ Evaluate" cycle.) At the beginning, designers analyze questions or themes in order to get problem-solving clues. Furthermore, they integrate related experience and data within database to get stage results and then determine suitability by evaluate results. Design is gradually forming under the repeated adjustment of loop. (FIGURE 4) 
“Image $\rightarrow$ Present $\rightarrow$ Test" $\quad$ (Zeisel, 1981) : This design process is composed under the helix structure of three loops. Along with design process, the range and topic will become narrower and clearer. (FIGURE 5)

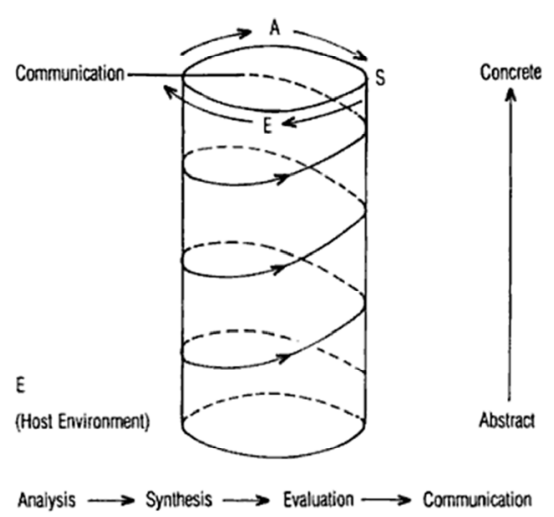

Figure 2. Asimow's model (Asimow, 1962)

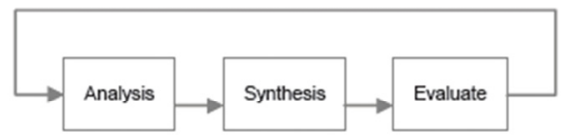

Figure 4. Alexander's model (Alexander, 1964)

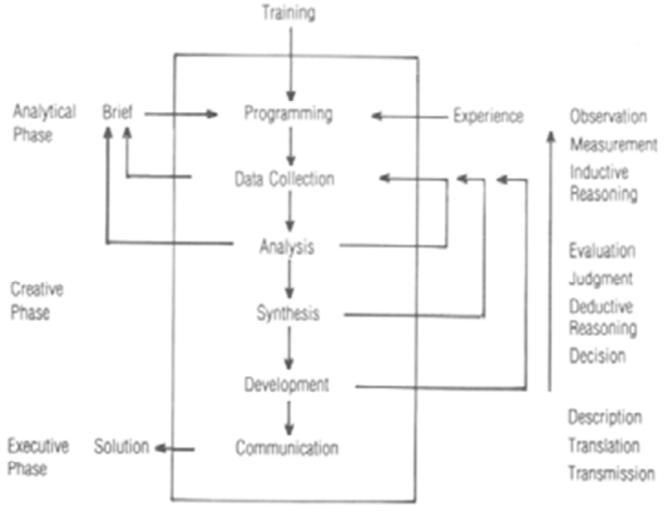

Figure 3. Archer 's model (Archer, 1965)

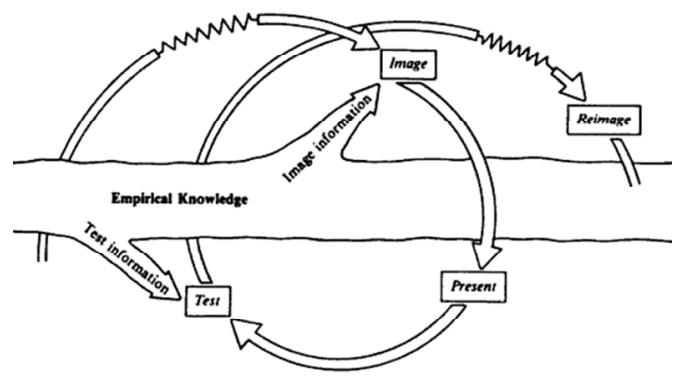

Figure 5. Zeisel's model (Zeisel, 1981)

"Programming $\rightarrow$ Design" (Pena, 1987) : It is the process similar to analyze first and then integrate. Designers are always making the plan before desig $\mathrm{n}$ and then follow it' s content.

\subsection{Design thinking}

Within exploration during design process and its model, design problem is an ill-define problem that has unclear problems and uncertain answers. That's why design research notices the structure and characteristic of design problem in order to solve the internal state of logic and decision-making process. From the perspective of problem-solving behavior and the basis of knowledge, Newell, Shaw \& Simon (1957, 1967) brought up problem-solving information processing theory and indicated that thinking is a process of information operation. The description and analysis of the process of human cognition explain the relationship between action and cognition.

Model 1. Search model (Simon, 1969; Rowe, 1987) :

Based on problem-solving and data-processing, design can be seen as a procedure of strategy-searching. The representation of problem space and the state of knowledge is "Decision Tree" (FIGURE 6) which shows whole possibilities. Designers can make decisionmaking process easier by using preference and simplifying assumptions. Design circumstance reveals change tendency of trial-and-error and generate-and-test.

Model 2. $\ulcorner$ Problem $\rightarrow$ Solving $\lrcorner($ Munari, 1981) $:$

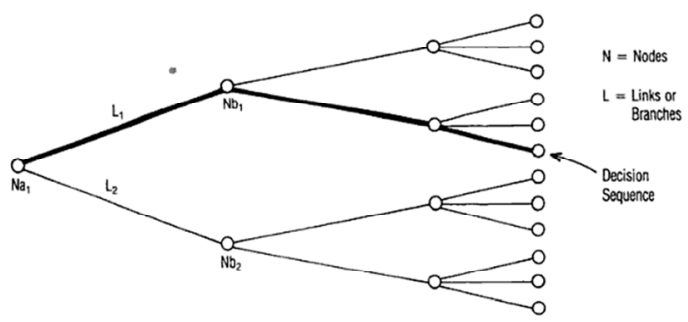

Figure 6. Decision tree (after Rowe, 1987, p.53)

In short, although the design problem only has one unit, it can be solved by many factors or methods. Thus the most important way is to understand problems and seek solutions. 
Model 3. $\ulcorner$ Problem-seeking $\rightarrow$ Problem-solving $\lrcorner($ Pena, 1987) :

Designers under the situation of "Problem-seeking $\rightarrow$ Problem-solving" try to find out and define problem for themselves.

\subsection{State Space}

The structure of State Space is often used to explain the model of design process among design computing area and each design phase can be regarded as every stand-alone status. The connection from state to state represents the designer's operation and resembles effects that change the status of certain properties. In one word, each state is a node in the space and the conjunctions between each node are lines in the space.

As we regard design process as a state space, fragments of state-recording contain thinking process of design. While we connect every action as continuous design process, large amount of design data will be valued.

State space is a simple model is used to describe machine's status and adjustment in computer science domain. In the field of mathematics, we can use a value group as " $\mathrm{N}, \mathrm{A}, \mathrm{S}, \mathrm{G}$ ". " $\mathrm{N}$ " means collection of states. "A" indicates connection of states. " $\mathrm{S}$ " is the beginning status of non-hollow conjunction. "G" presents status of non-hollow target (W.-T. Chang, 2006). State space is always used to explain the model of design process in the field of design computing, therefore, each phase of design step can be seen as a standalone status. The connection from state to state represents the designer's operation and resembles effects that change the status of certain properties. In one word, each state is a node in the space and the conjunctions between each node are lines in the space. By way of this, it comprises a state space that can describe and solve design process problems.

\section{RESEARCH METHOD}

This research builds a computer aid design system prototype that can generate watch design automatically by the rule of Genetic Algorithms. The system decodes basic forms of a watch and then encodes by forms and texture. After encoding, the system generates geometric watch forms by way of crossover of Genetic Algorithms

\subsection{System framework}

The system disassembles the form of watch into basic geometric forms as the data base. Select elements from data base and generate design by crossover of the Genetic Algorithms of Rule Base. The result can crossover, save and generate again. (figure 7).

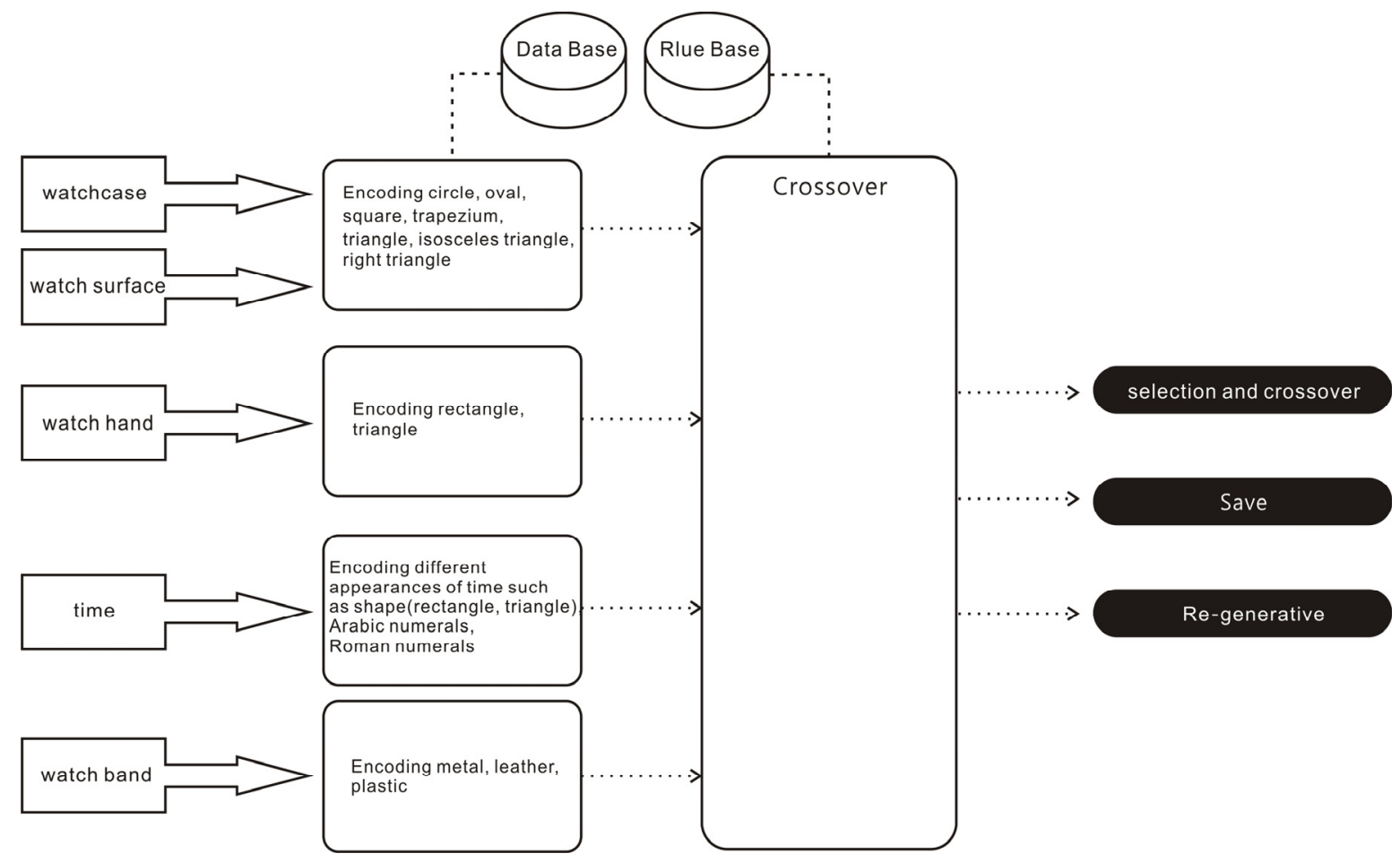

Figure 7. System framework 


\subsection{Genetic Algorithms}

Representation :

The watch is been take apart into fine parts-A: watchcase, $B$ : watch surface, $C$ : watch band, D: watch hand, E: time(FIGURE 8)

Encode :

Among the watchcase and watch surface that compose of basic geometric form, the circle, square, triangle are being used most often. Then, they are transformed into circle, oval, square, trapezium, triangle, isosceles triangle, right

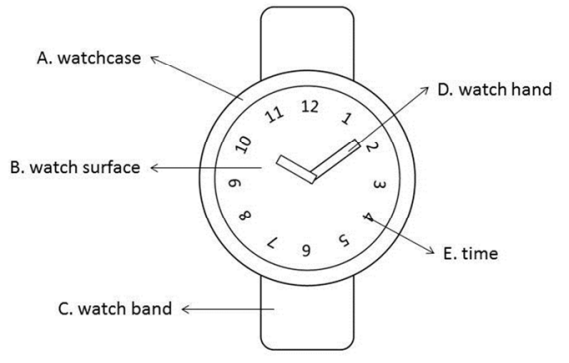

Figure 8. Representation of the watch triangle and create the form of watchcase and watch surface.

Watchcase encoding:

Encoding circle, oval, square, trapezium, triangle, isosceles triangle, right triangle as A1 、 A2 、 A3 、 A4 、 A5 、 A6 - A7 、 A8.

Watch surface encoding:

Encoding circle, oval, square, trapezium, triangle, isosceles triangle, right triangle as B1 、 B2 、 B3 、 B4 、 B5 、 B6 $、$ B7 、 B8.

Watch band

Encoding metal, leather, plastic as $\mathrm{C} 1 \cdot \mathrm{C} 2 、 \mathrm{C} 3$.

Form of watch hand:

Encoding rectangle, triangle as D1 D2.

Time:

Encoding different appearances of time such as shape(rectangle, triangle), Arabic numerals, Roman numerals as E1 VE2 、 E3 、 E4.

Crossover :

The form of watch is created by way of crossover A1 、 A2 、 A3 、 $\mathrm{A} 4 、 \mathrm{~A} 5 、 \mathrm{~A} 6 、 \mathrm{~A} 7 、 \mathrm{~A} 8, \mathrm{~B} 1 、 \mathrm{~B} 2 、 \mathrm{~B} 3 、 \mathrm{~B} 4 、 \mathrm{~B} 5 、 \mathrm{~B} 6 、 \mathrm{~B} 7 、$ $\mathrm{B} 8, \mathrm{C} 1 、 \mathrm{C} 2 、 \mathrm{C} 3, \mathrm{D} 1 、 \mathrm{D} 2, \mathrm{E} 1 、 \mathrm{E} 2 、 \mathrm{E} 3 \cdot \mathrm{E} 4$ of the Genetic Algorithms(FIGURE 9)

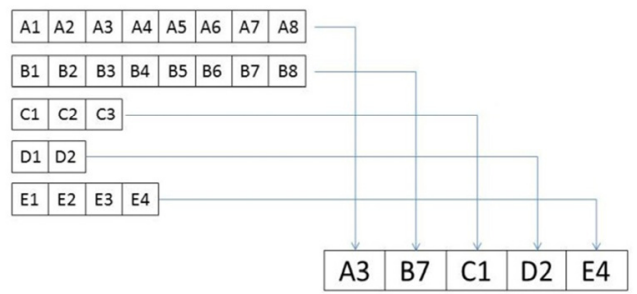

Figure 9. the form of watch is created by way of crossover

\section{RESULTS}

\subsection{Prototype system}

This research builds a computer aid design prototype that can generate watch design automatically by the rule of the Genetic Algorithms. The system disassembles the form of watch into basic geometric forms as the data base. Select elements from data base and generate design by crossover of the Genetic Algorithms of Rule Base. The result can crossover, save and generate again(FIGURE 10).

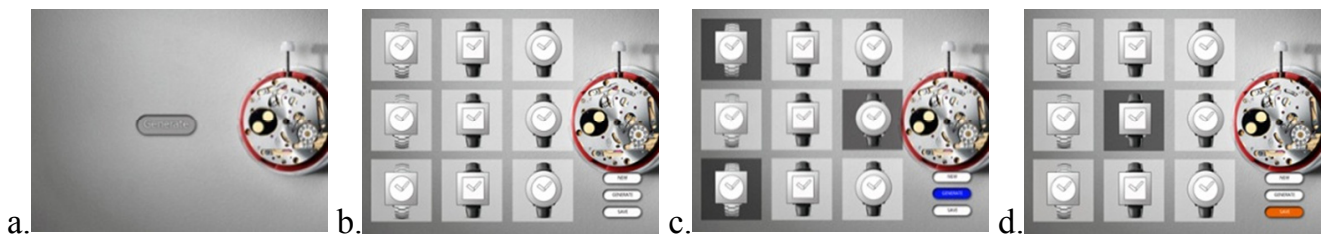

Figure 10. a. Start ,b. Generative, c.Selection, d.Save 


\subsection{Dynamic graphic}

The research expands generative design of watch by way of the operation of the prototype. By this system, the research collects data during design process and visualizes the design process to bring dynamic graphic by STATE SPACE SEARCH. (FIGURE 11).

\section{DISCUSSION}

The watch design prototype system that builds in this research can decode, encode and generate the design of watch. Therefore, it can create large numbers of design in a short time. By collecting date to bring out the developed dynamic graphic during design process base on the State Space. Moreover, designers can use the system to choose the right form and generate again. Not only designers can simplify the process of concerning the combinations of possible forms during design process, but also reduce time

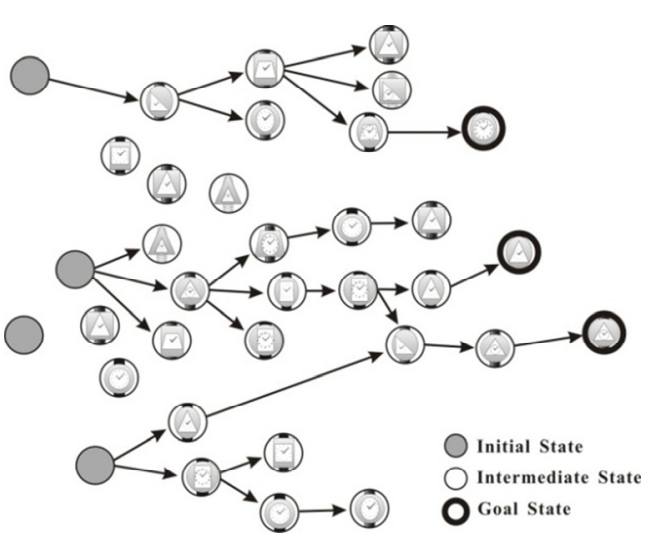

Figure 11. Dynamic graphic consuming and raise the design efficiency.

By way of visualizes design process by dynamic graphics that produce from watch generative design process, the research believes dynamic graphics can help to record data that leaping among status during design process. It helps designer clearly observes form-transforming details during design process. Through dynamic graphics, not only the designer can keep the final status record, but also thinks over some useful data (such as transform between two forms or some in-development idea) that might lost during design process after design is finished. Those recorded data will help designers to review design process as well as provide certain recorded node to support designers develop images un the future.

\section{REFERENCES}

Wassily Kandinsky. Point and Line to Plane. Dover Publications, New York.

John Holland, "Adaptation in Natural and Artificial Systems," Ann Arbor Univ. of Michigan Press, 1975.

Woodbury, R. F., "Searching for design; Paradigm and practice", Building and Environment, vol. 26, pp. 6173, 1991.E.R. Tufte. Envisioning Information. Cheshire, CT, Graphics Press. 1990.

Dan Braha and Oded Maimon. A mathematical Theory of Design:Foundations,Algorithms and Applications. 1998.

Teng-Wen Chang. Geometric Typed Feature Structures:Toward design space exploration. 1999.

Newell, A., Shaw, J. C., \& Simon, H. A., (1967). The process of creative thinking. In H. Gruber, G. Terrell, \& M. Wertheimer(eds.),Contemporary Approaches to Creative Thinking, New York: Atherton Press.

Gero, J., \& Tang, H. H. (2001). The differences between retrospective and concurrent protocols in revealing the process-oriented aspects of the design process. Design Studies, 22, 283e295.

Woodbury, R. F., "Searching for design; Paradigm and practice", Building and Environment, vol. 26, pp. 6173, 1991.E.R. Tufte. Envisioning Information. Cheshire, CT, Graphics Press. 1990

Teng-Wen Chang. Geometric Typed Feature Structures:Toward design space exploration. 1999

Eckersley, M. 1988. "The form of design process: A protocol analysis study.” Design Studies. 16(2), 86-94.

Ericsson, K. A. \& Simon, H. A. 1993. Protocol analysis-Verbal Report as Data. Revised ed., Cambridge, MA: MIT Press. 